\title{
PERFIL DAS MULHERES USUÁRIAS DO SUS COM LESÕES INTRAEPITELIAIS EM UM MUNICÍPIO DO OESTE DO PARANÁ
}

\author{
Tatiane Rafaelle Moreira*1 \\ Aline Cristine Soares de Lima ${ }^{1}$ \\ Maiara Aline dos Santos ${ }^{2}$ \\ Marcos Ereno Auler ${ }^{3}$ \\ Maurício Turkiewicz ${ }^{4}$ \\ Michele Ana Flores Chaves ${ }^{5}$ \\ Jacqueline Plewka ${ }^{5}$
}

MOREIRA, T. R.; LIMA, A. C. S. de; SANTOS, M. A. dos; TURKIEWICZ, M.; CHAVES, M. A. F.; PLEWKA, J. Perfil das mulheres usuárias do sus com lesões intraepiteliais em um município do oeste do Paraná. Arq. Cienc. Saúde UNIPAR, Umuarama, v. 21, n. 3, p, 181-186, set./dez. 2017.

\begin{abstract}
RESUMO: O câncer cervical é o quarto tipo de tumor mais frequente entre mulheres e quarta causa de mortalidade feminina por câncer no Brasil. Neste trabalho o objetivo foi demostrar o perfil das mulheres atendidas pelo SUS que apresentaram lesões intraepiteliais de baixo grau (LSIL) e alto grau (HSIL) no exame preventivo citopatológico realizado por um laboratório de citopatologia credenciado ao SUS que atende Cascavel-PR e distritos. No período de maio de 2014 a maio de 2015 foram observados 395 exames citopatológicos com LSIL e 160 com HSIL, cuja faixa etária predominante foi dos 25 aos 64 anos (70,64\%). Em média observou-se os seguintes resultados: a JEC esteve presente em $90,56 \%$ das amostras; o colo cervical apresentou-se normal em 77,31\% das mulheres e 43,58\% destas mulheres em estudo haviam realizado um exame anterior em até um ano, sendo que o maior motivo para realização do mesmo foi o rastreamento (89,11\%). Mais que $90 \%$ das mulheres não apresentaram sangramentos, seja após a relação sexual ou menopausa. As lesões também prevaleceram em mulheres atendidas na UBS (61,98\%) mesmo elas sendo em menor número se comparadas àquelas atendidas por USF. Esses dados demostram a importância da realização desse exame, pois na maioria das lesões as mulheres não apresentaram sintomas ou alterações clínicas. PALAVRAS-CHAVE: Câncer do colo do útero. Citopatológico cervical. Exame de Papanicolaou. Exame preventivo do câncer do colo do útero. Lesão intraepitelial de alto grau. Lesão intraepitelial de baixo grau.
\end{abstract}

\section{PROFILE OF FEMALE USERS OF THE BRAZILIAN UNIFIED HEALTH SYSTEM WITH INTRAEPITHELIAL LESIONS IN A CITY IN WESTERN PARANA}

\begin{abstract}
Cervical cancer is the fourth most frequent kind of cancer among women and the fourth cause of female mortality due to cancer in Brazil. The purpose of this work was to present the profile of women attended by the Brazilian Unified Health System (SUS) who showed low-grade (LSIL) and high-grade (HSIL) intraepithelial lesions at the cytopathology screening test performed by a SUSaccredited cytopathology laboratory serving the city of Cascavel-PR and its districts. In the period between May 2014 and May 2015, a total of 395 cytopathologic test presenting LSIL and 160 with HSIL were observed. The age group ranged from 25 to 64 years old (70.64\%). The following average results were observed: squamocolumnar junction was present in $90.56 \%$ of the samples; normal cervix in $77.31 \%$ of women and $43.58 \%$ of the women in the study had already had an examination within the previous year, with the main reason for the present exam was screening (89.11\%). More than $90 \%$ of women did not present any bleeding, either after sexual intercourse or menopause. The lesions also prevailed in women attending primary health care ( $61.98 \%$ of lesions) even though they were in lower number when compared to those served by family health programs. Such information demonstrates the importance of carrying out this examination, since most lesions are asymptomatic or do not show any clinical changes.
\end{abstract}

KEYWORDS: Cervical cancer. Cervical cytology. High-grade intraepithelial lesion. Low-grade intraepithelial lesion. Pap test. Screening for cervical cancer.

\section{Introdução}

O câncer é uma doença de distribuição mundial, sendo um problema de saúde pública, principalmente em países em desenvolvimento. No mundo, os tipos de tumores mais frequentes são pulmão, mama, intestino e próstata. $\mathrm{O}$ câncer do colo do útero é o quarto mais frequente entre as mulheres no mundo, com 530.000 novos casos em 2012, representando cerca de 7,5\% dos óbitos das mulheres por este câncer neste ano. Com uma estimativa de mais de 270 mil óbitos a cada ano, a maior parte dos casos (85\%) se concentra em regiões menos desenvolvidas (WHO, 2016; INCA,
2015).

O câncer do colo do útero, também chamado de câncer cervical, é a quarta causa de mortalidade feminina por câncer no Brasil, com 5.430 óbitos em 2013 (INCA, 2015). Estimam-se no Brasil para 2016, mais de 16.000 novos casos deste câncer, com um risco estimado de 15,85 casos a cada 100.0000 mulheres, sendo o tipo de tumor mais incidente entre as mulheres na região norte $(23,97$ para cada 100.000 mulheres), o segundo mais incidente nas regiões centro-oeste $(20,72$ para 100.000$)$ e nordeste $(19,49$ para 100.000$)$, o terceiro na região sudeste $(11,30$ para 100.000$)$ e o quarto na região sul (15,17 para 100.000) (BRASIL, 2015). Em 2013 a

DOI: 10.25110/arqsaude.v21i3.2017.6181

${ }^{1}$ Acadêmica do Programa de Residência Farmacêutica em Análises Clínicas no Hospital Universitário do Oeste do Paraná, Universidade Estadual do Oeste do Paraná, Cascavel - PR, Brasil. Av. Tancredo Neves nº 3224, Bairro Santo Onofre, CEP 85.806-470, Cascavel - PR, Brasil.*E-mail: tatimoreira18@yahoo. com.br

${ }^{2}$ Estagiária no Labcell Citologia Diagnóstica LTDA, Cascavel - PR, Brasil

${ }^{3}$ Docente do curso de Farmácia na Universidade Estadual do Centro Oeste do Paraná, Guarapuava - PR, Brasil

${ }^{4}$ Diretor técnico no Labcell Citologia Diagnóstica LTDA, Cascavel - PR, Brasil

${ }^{5}$ Docente do curso de Farmácia e do Programa de Residência Farmacêutica em Análises Clínicas na Universidade Estadual do Oeste do Paraná, Cascavel - PR, Brasil 
taxa de mortalidade bruta por câncer do colo de útero no Brasil foi de 5,49 para cada 100.000 mulheres, no Paraná neste mesmo ano esta taxa bruta foi de 5,41 para cada 100.000 mulheres, e em Cascavel também em 2013 a taxa bruta foi de 10,03 para cada 100.000 mulheres (INCA, 2015).

O principal causador desta doença é a infecção persistente pelo Papilomavírus Humano (HPV), esse vírus é transmitido por meio de contato íntimo, e pode atingir tanto homens quanto mulheres, sendo que na maioria das vezes a infecção cursa de forma assintomática, e regride espontaneamente sem necessidade de qualquer intervenção. Existem mais de 100 tipos de vírus HPV, sendo que os tipos 16 e 18, que possuem alto potencial oncogênico, são os responsáveis por aproximadamente $70 \%$ dos casos do câncer do colo do útero. Além da infecção deste vírus, existem outros fatores que podem contribuir com a evolução dessa doença como: tabagismo, iniciação sexual precoce, múltiplos parceiros sexuais, multiparidade, imunossupressão e o uso de anticoncepcionais orais (WHO, 2016).

Como essa patologia apresenta uma evolução lenta, cerca de 10 anos para se desenvolver, o Ministério da Saúde (MS) prioriza a realização do exame citopatológico cervical, em mulheres a partir dos 25 anos (ou que já tenham iniciado a atividade sexual) até os 64 anos, faixa etária denominada população alvo para o rastreamento deste câncer (BRASIL, 2011).

Em 2011 foi lançado no Brasil o Plano de Fortalecimento da Rede de Prevenção, Diagnóstico e Tratamento do Câncer, sendo citado entre eles o câncer cervical. Entre os objetivos estão desde assegurar o acesso ao exame preventivo até o tratamento das lesões (INCA, 2015; BRASIL, 2013). A principal ferramenta utilizada atualmente na prevenção do câncer cervical é o citopatológico oncótico, o Papanicolaou, que é um exame de fácil disponibilidade sendo ofertado também em postos ou unidades de saúde da rede pública e detecta lesões precursoras do câncer do colo do útero (INCA, 2015).

Conforme a Nomenclatura Brasileira para Laudos Citopatológicos Cervicais (NBLCC), as alterações citopatológicos observadas nos exames preventivos são denominadas de células atípicas de significado indeterminado, e atipias em células escamosas e glandulares (BRASIL, 2012). Dentre as alterações celulares presentes nas atipias de células escamosas, as alterações mais frequentes observadas no Brasil são as lesões intraepiteliais de baixo grau (LSIL) e as lesões intraepiteliais de alto grau (HSIL) (BRASIL, 2012).

Portanto, este trabalho objetiva apresentar o perfil das mulheres atendidas pelo Sistema Único de Saúde (SUS) em uma cidade do oeste do Paraná com resultados dos exames citopatológicos do colo do útero com lesões intraepiteliais de baixo grau e alto grau, bem como a prevalência dessas lesões intraepiteliais. Segundo informações do Instituto Nacional do Câncer estas lesões somam aproximadamente $40 \%$ ou mais dos exames alterados avaliados no país, sendo que HSIL apresenta maior possibilidade de evoluir para um carcinoma.

\section{Metodologia}

Foi realizado um estudo retrospectivo, com a busca dos dados presentes nas requisições dos exames citopatológicos do colo do útero, das mulheres atendidas pelo Laborató- rio Labcell, credenciado ao SUS no município de Cascavel-PR e distritos, no período de maio de 2014 a maio de 2015 , este trabalho foi desenvolvido após a aprovação pelo comitê de ética em pesquisa envolvendo seres humanos da Universidade Estadual do Oeste do Paraná (UNIOESTE), com parecer número 1.180.887.

Nesse trabalho foram avaliadas 1036 requisições citopatológicas com resultados citológicos alterados conforme a Nomenclatura Brasileira para Laudos Citopatológicos (BRASIL, 2012). Foram incluídas nesse trabalho 555 exames citopatológicos com resultados de atipias em células escamosas classificadas como LSIL e HSIL, sendo que as demais alterações citopatológicas foram excluídas.

Os exames citopatológicos incluídos neste estudo foram provenientes das seguintes unidades de saúde: 16 Unidades Básicas de Saúde (UBS); 20 Unidades Saúde da Família (USF); e 04 outras unidades como: Universidade Paranaense (UNIPAR), do Hospital Universitário do Oeste do Paraná (HUOP); do Centro Especializado de Doenças Infecto Parasitárias (CEDIP) e do Consórcio Intermunicipal de Saúde do Oeste do Paraná (CISOP).

As variáveis deste trabalho foram: grupo etário (abaixo de 25, 25 a 64 anos e acima de 64 anos); presença da junção escamocolunar (JEC) e/ou zona de transformação (ZT); o motivo da realização deste exame (rastreamento, repetição e seguimento); o período do último exame preventivo (nunca fez o exame citopatológico, fez no ano anterior, ou dois a mais anos anteriores); presença de sangramento após relação sexual ou menopausa; bem como as informações referentes à inspeção do colo do útero durante o exame clínico (colo normal, ausente, alterado e não visualizado). Os dados foram avaliados e agrupados na planilha do Microsoft Excel $^{\circledR}$.

\section{Resultados}

Dos 1036 exames citopatológicas com alterações citopatológicas avaliados entre maio de 2014 a maio de 2015, $555(53,57 \%)$ apresentaram LSIL e HSIL, sendo que 395 (38,13\%) foram LSIL, cujas idades variaram entre 15 a 80 anos (média de 47,5 anos), e 160 (15,44\%) HSIL com idades entre 16 a 74 anos (média de 45 anos), como apresentado na Figura 1.

Figura 1: Distribuição de LSIL e HSIL por grupo etário no município de Cascavel-PR e distritos

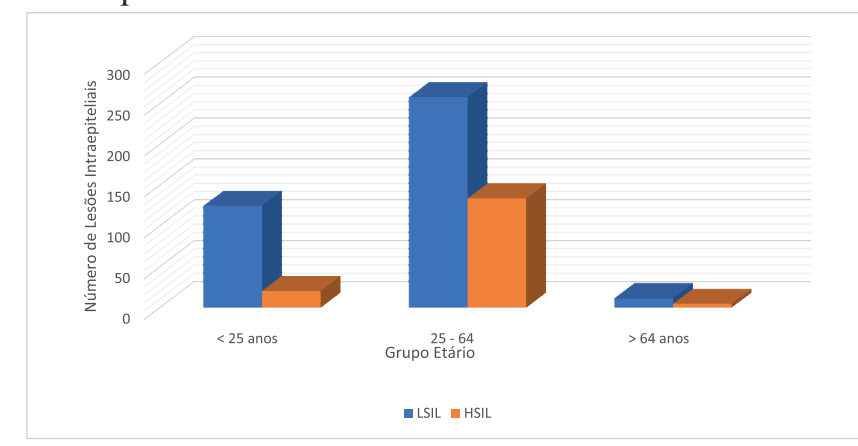

No grupo etário dos 25 aos 64 anos, houve o predomínio das lesões sendo 258 mulheres com LSIL (46,50\%) e 134 com HSL (24,14\%). Entre as mulheres abaixo dos 25 anos, 125 delas foram classificadas com LSIL (22,52\%) e 
21 com HSIL (3,78\%). Já entre aquelas maiores de 64 anos, 12 foram classificadas com LSIL $(2,16 \%)$ e 5 com HSIL $(0,90 \%)$. Em relação a representação da JEC e/ou ZT dos exames avaliados, esses foram representativos em 355 (89,87\%) dos exames com LSIL, e em 146 (91,25\%) com HSIL.

Conforme os dados da Figura 2, apesar deste município apresentar mais USF (20 unidades) do que UBS (16 unidades), as lesões intraepiteliais foram mais frequentes em mulheres atendidas na UBS $(61,98 \%$ das lesões) das quais 244 apresentaram LSIL e 100 foram classificadas como HSIL ao exame citopatolágico cervical, sendo que nas USF's foram 121 mulheres com LSIL e 45 com HSIL (29,91\% das lesões). Os demais atendimentos como HUOP, CISOP, CEDIP e UNIPAR compreenderam $8,11 \%$ de lesões intraepiteliais das quais 30 foram LSIL e 15 HSIL.

Figura 2: Distribuição de LSIL e HSIL por unidade de saúde do município de Cascavel-PR e distritos

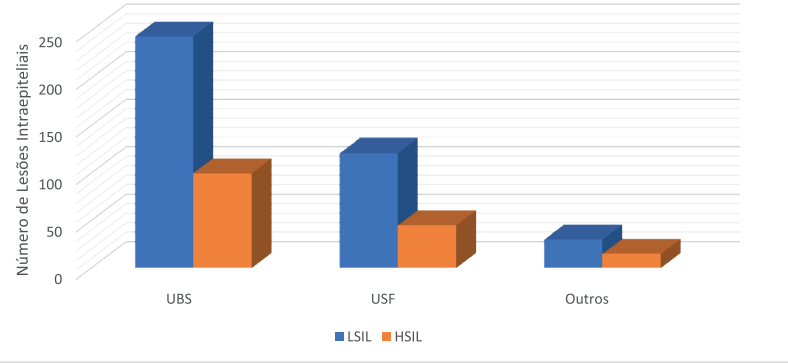

A Tabela 1 demonstra as informações referentes aos dados da anamnese presentes na requisição do exame citopatológico do colo do útero, do Programa Nacional de Controle do Câncer do Colo do Útero do MS. Em 129 (23,24\%) das requisições avaliadas não houve o preenchimento dessas informações. Já em relação à periocidade da realização anterior do exame citopatológico do colo do útero, a maioria das mulheres com LSIL realizaram seu preventivo com intervalo menor ou igual a 1 ano (42,78\%), seguido de não informado (NI) e 2 anos anteriores com percentuais equivalentes (16,46\% e $16,20 \%$ respectivamente).

Nas mulheres com HSIL, a maioria realizou seu exame preventivo em até 1 ano $(44,38 \%)$, seguido de NI $(18,12 \%)$, e 2 anos anteriores $(16,88 \%)$. Em relação ao motivo de realização do exame relatado nas requisições, predominou-se o rastreamento, ou seja, aquelas mulheres que estão realizando o exame como rotina do programa de controle do câncer de colo uterino em ambas as lesões, em 87,60\% das mulheres com LSIL, e 90,62\% com HSIL. A repetição e o seguimento dessas lesões, conforme preconizado pelo MS e INCA, tiveram percentuais próximos como segue: $5,82 \%$ e $1,77 \%$ em LSIL; e 5,62\% e 1,88\% em HSIL respectivamente (Tabela 1).

Neste trabalho, a maioria das mulheres tanto com LSIL $(88,87 \%)$ quanto com HSIL $(91,87 \%)$ não relataram nenhum tipo de sangramento. Em relação ao sangramento após a relação sexual, as frequências nas duas lesões apresentaram-se próximas $(7,59 \%$ e $6,25 \%$ respectivamente). A figura 3 apresenta as informações referentes a inspeção do colo do útero durante o exame citopatológico, e apesar desse estudo avaliar somente os dados referentes as mulheres com lesões intraepiteliais, o colo do útero apresentou-se normal durante a coleta do exame preventivo na maioria das lesões, com frequências muito próximas entre as lesões, sendo 312 mulheres com LSIL $(78,99 \%)$ e 121 daquelas com HSIL $(75,62 \%)$, enquanto que a visualização alterada do colo do útero alterado, ocorreu em apenas 57 das mulheres com LSIL $(14,43 \%)$ e 26 daquelas com HSIL (16,25\%).

Tabela 1: Dados da anamnese da requisição de exame citopatológico do colo do útero

\begin{tabular}{c|cc|cc}
\hline Informações & \multicolumn{2}{c|}{ LSIL } & \multicolumn{2}{c}{ HSIL } \\
\hline Período de Realização & N & $\mathbf{( \% )}$ & $\mathbf{N}$ & $\mathbf{( \% )}$ \\
Até 1 ano & 169 & 42,78 & 71 & 44,38 \\
2 anos & 64 & 16,20 & 27 & 16,88 \\
$\geq 3$ anos & 44 & 11,14 & 22 & 13,75 \\
$1^{\circ}$ exame & 53 & 13,42 & 11 & 6,87 \\
NI & 65 & 16,46 & 29 & 18,12 \\
Motivo da Realização & & & & \\
Rastreamento & 346 & 87,60 & 145 & 90,62 \\
Repetição & 23 & 5,82 & 9 & 5,62 \\
Seguimento & 7 & 1,77 & 3 & 1,88 \\
NI & 19 & 4,81 & 3 & 1,88 \\
Presença de Sangramento & & & & \\
Ausente & 351 & 88,87 & 147 & 91,87 \\
Após Relação Sexual & 30 & 7,59 & 10 & 6,25 \\
Após Menopausa & 4 & 1,01 & 0 & 0,00 \\
NI & 10 & 2,53 & 3 & 1,88 \\
\hline
\end{tabular}

NI: Não informado na requisição

Figura 3: Distribuição de LSIL e HSIL com as informações da inspeção do colo uterino no exame clínico.

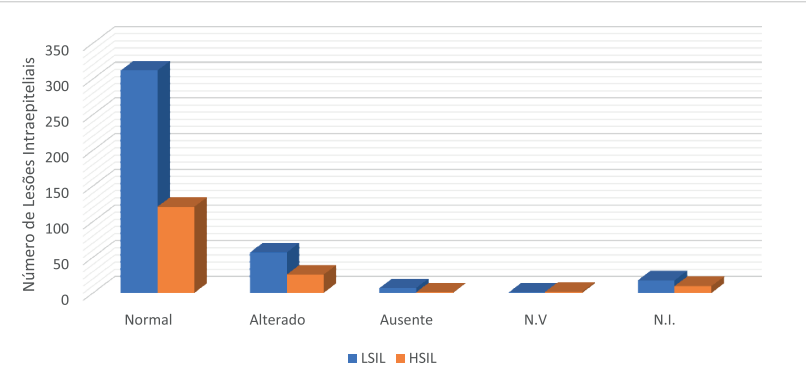

N.I.: Não Informado na requisição. N.V.: Não Visualizado

\section{Discussão}

Em estudo realizado em Erechim-RS no período de 2007 a 2011, em 2.049 exames citopatológicos alterados, $53,39 \%$ corresponderam a LSIL e $8,74 \%$ HSIL (BACKERS et al, 2014). Em Natal-RN, entre 2005 e 2010, das 134 mulheres que apresentaram alterações no exame citopatológico, $73,1 \%$ foram LSIL e 3,7\% HSIL (LAGANÁ et al., 2013). Portanto, conforme os dados destes trabalhos, o percentual de lesões intraepiteliais tanto de baixo grau quanto de alto grau demonstram uma variação na população estudada, o que demostra a importância do perfil epidemiológico da população, para que esses dados possam contribuir para as campanhas de prevenção do câncer do colo do útero.

Conforme dados do MS/INCA (BRASIL, 2011), no Brasil entre todas as alterações citopatológicas, a LSIL foi a segunda alteração mais frequente $(31 \%)$, percentual próximo ao observado em nosso estudo $(38,13 \%)$. Isso pode ser justificado, porque essa lesão também engloba os efeitos citopatológicos do vírus HPV. Ainda em relação a esses dados 
de 2011, foram visualizadas HSIL em 9,7\% dos exames alterados no país. Portanto, em nosso trabalho HSIL apresentou uma frequência maior que a observada no Brasil naquele ano, o que pode refletir no percentual dos exames compatíveis com HSIL dos indicadores do Monitoramento Interno de Qualidade (MIQ).

Os resultados dos exames citopatológicos compatíveis com HSIL influenciam os seguintes indicadores do MIQ: índice de positividade; percentual de exames compatíveis com lesão intraepitelial de alto grau; percentual de atipias escamosas entre os exames alterados e razão entre atipias escamosas de significado indeterminado e lesões intraepiteliais escamosas. Portanto, quando esses indicadores estão abaixo do recomendado podem indicar a dificuldade de interpretação dos critérios citomorfológicos observados, e portanto pode significar que essas lesões não estão sendo detectadas na população em estudo sendo que devido a elevada capacidade de progressão da lesão de alto grau ao câncer cervical, estas lesões são o alvo da campanha de prevenção do câncer do colo do útero (BORTOLON et al., 2012).

Uma amostra com boa qualidade para análise oncótica deve apresentar células em quantidade representativa do colo do útero, bem distribuídas, fixadas e coradas, que permita uma conclusão diagnóstica (BRASIL, 2011). A representação dos epitélios da JEC e/ou ZT é importante no exame citopatológico cervical, pois é nessa região que se localizam mais de 90\% (BRASIL, 2013) das lesões precursoras malignas do colo do útero; portanto essa representação influencia a sensibilidade do exame citopatológico, conforme demostrado nesse estudo com presença da JEC e/ou ZT em média de $90,56 \%$ nas lesões avaliadas refletindo assim a qualidade dos exames citopatológicos.

No Brasil em 2013, a média da representação da ZT em todas as faixas etárias avaliadas dos exames citopatológicos avaliados foi $55,8 \%$ (BRASIL, 2015). Em outro trabalho desenvolvido no Maranhão, 64,2\% das amostras citopatológicas apresentaram tais epitélios (SILVA et al., 2014), portanto percentuais abaixo do observado nesse trabalho (90,56\%). $\mathrm{O}$ treinamento dos profissionais que atuam na prevenção do câncer cervical é de extrema importância, pois a qualidade da coleta do material cervical reflete no diagnóstico do exame citopatológico (MANRIQUE et al., 2009).

Nesse trabalho a maioria das lesões intraepiteliais foram evidenciadas no grupo etário da população alvo da campanha de prevenção contra o câncer do colo do útero do MS e INCA, porém os dados também demonstram a importância da realização do exame citopatológico do colo do útero em mulheres mais jovens, que já iniciaram a atividade sexual, pois evidenciou-se neste trabalho 125 exames com LSIL $(22,52 \%)$ e 21 com HSIL $(3,78 \%)$ em mulheres com até 24 anos.

Prado et al. (2012) relatam em trabalho desenvolvido no Rio Branco-AC que dos 846 exames alterados encontrados entre os anos de 2007 e 2008, 191 (22,6\%) eram de mulheres abaixo dos 25 anos, e a maioria delas iniciaram a vida sexual antes dos 18 anos, sendo que 30,5\% iniciaram antes dos 14 aos, e 50,8\% entre 15 e 17 anos.

Em um trabalho realizado com 198 mulheres com infecção por HPV atendidas pelo SUS, em um Hospital de Montes Claros-MG, 132 (66,7\%) tinham entre 20 e 39 anos, e tanto nas pacientes que apresentaram LSIL como HSIL, a maior parte $(61,6 \%)$ iniciou a vida sexual antes dos 20 anos.
Dessa forma, no trabalho de Silva, Coelho e Athayde (2016), ressaltam a hipótese da adequação da faixa etária alvo da prevenção deste câncer pelo MS/INCA, considerando o início da atividade sexual cada vez mais cedo no Brasil.

Conforme os percentuais apresentados, o USF tem quase a metade do percentual de lesões alteradas em relação a UBS (29,91\% e 61,98\% respectivamente), no município de Cascavel-PR. Isso pode ser justificado pelo fato que as USF são unidades de saúde que trabalham com a Estratégia da Saúde da Família (ESF), modelo proposto pelo MS no qual o foco é o atendimento integral ao indivíduo, família e comunidade. Portanto, a equipe multidisciplinar cadastra e acompanha as famílias por meio de visitas domiciliares na área de sua abrangência, e com esse cadastro e as informações coletadas é possível conhecer a realidade daquela população (FIGUEREDO, 2012; SESAU, 2015). Já as UBS são chamadas de "porta de entrada" para o SUS, com estabelecimentos de atenção primária, cujo objetivo é atender $80 \%$ dos casos de saúde sem a necessidade de encaminhamento hospitalar (PBDA, 2016). São unidades que desenvolvem atividades assistenciais e de prevenção em seu território da abrangência, e responsabilizam-se pelo atendimento ambulatorial programado nas áreas de saúde (SESAU, 2015).

Em um estudo desenvolvido em duas USF com ESF em Minas Gerais, onde foi avaliado o trabalho da equipe multidisciplinar no combate ao câncer do colo do útero, a mesma descreveu que a principal etapa na prevenção dessa patologia, é a realização do exame preventivo junto com a busca ativa da população, onde a equipe orienta as mulheres que não estão comparecendo aos exames, ou que não estão dando continuidade ao tratamento (OLIVEIRA et al, 2010). De acordo com a Organização Mundial de Saúde (OMS), com uma cobertura da população alvo, de no mínimo $80 \%$, com a garantia de diagnóstico e tratamento adequados, a incidência do câncer cervical pode ser reduzida em até $90 \%$, e quando essa doença é descoberta no início, as chances de cura podem chegar a 100\% (INCA, 2015; INCA, 2016).

Observou-se também a ausência de preenchimento de informações em $23,24 \%$ dos dados das requisições do exame citopatológico cervical, os quais são de preenchimento obrigatório pela equipe de saúde responsável pela coleta do exame, sendo que essas são de extrema importância para a correlação clínica laboratorial da lesão (regressão ou progressão), ou para o encaminhamento do exame para o monitoramento externo de qualidade previsto no Manual de Gestão de Qualidade para Laudos Citopatológicos (BRASIL, 2012).

Segundo os fluxogramas das recomendações preconizadas para as condutas clínicas pelo MS/INCA, a mulher deve repetir seu exame preventivo em tempos variáveis conforme a faixa etária, com variação de repetição de 6 a 12 meses. Portanto, justifica essa frequência da realização do exame preventivo anterior em intervalo de até 1 ano evidenciado nesse trabalho. Já em relação às mulheres que apresentaram HSIL ao exame citopatológico cervical, relatamos acima que a maioria realizou o último exame preventivo em até 1 ano, sendo que a conduta preconizada pelo MS/INCA nessa lesão é a realização da colposcopia e quando confirmada a alteração, segue para biópsia, sendo que a repetição do exame citopatológico nas lesões precursoras pode ocasionar gastos desnecessários ao SUS, ou retardo no tratamento dessas lesões.

Outro dado evidenciado, é que $6,87 \%$ das mulhe- 
res com HSIL estavam realizando seu exame preventivo pela primeira vez, portanto demostra a importância das campanhas de prevenção dessa patologia, uma vez que a HSIL tem maior tendência para a progressão para o câncer cervical, considerada lesão altamente precursora desse câncer.

No relatório do INCA dos exames analisados em 2013, 46,9\% das mulheres realizaram o exame preventivo anterior em um intervalo de tempo menor ou igual a um ano (BRASIL, 2015), em nosso trabalho, apesar dos dados correspondem apenas aos exames com lesões intraepiteliais, também se observou uma frequência próxima aos dados do INCA (em média 43,58\%). No Boletim Informativo do MS/ INCA de 2014, mostra que na população alvo da campanha de prevenção deste câncer, 54\% das mulheres no Brasil, e $51 \%$ no Paraná, realizaram o exame preventivo em até um ano, portanto percentuais próximos ao observado em nosso estudo (BRASIL, 2014).

No trabalho de Rocha et al. (2012), realizado em um UBS de Santa Maria-RS, 70\% das mulheres entrevistadas tinham conhecimento do objetivo do exame preventivo, e a maioria referiu realizar o exame anualmente, porém, das que não o fazem, as principais queixas foram a dificuldade em marcar o exame, seja pela locomoção ou mesmo as longas filas de espera. Porém, em uma cidade do Ceará 66,67\% das mulheres entrevistadas mostraram nenhum conhecimento sobre o exame preventivo do câncer do colo do útero, sendo que muitas delas responderam que serve para detecção de doenças sexualmente transmissíveis, como a AIDS entre outras, e $60 \%$ relataram que procuram atendimento médico apenas quando possui algum sintoma como prurido, dor ou corrimento vaginal, mostrando que a informação e orientação sobre essa patologia tem grande impacto na prevenção (MOURA et al., 2010).

Os nossos dados demonstram que a maioria dos exames realizados com intervalo menor ou igual a 1 ano, não correspondem a repetição ou ao seguimento dessas lesões, conforme preconizado pela publicação do MS/INCA de 2011 (BRASIL, 2011), pois em média 89,11\% das mulheres com lesões intraepiteliais estão realizando seu preventivo como rotina.

Outro dado avaliado foi o predomínio da ausência de sangramento nas mulheres, em ambas as lesões intraepiteliais $(90,37 \%)$. Portanto, essa informação demonstra a importância da realização periódica dos exames preventivos do câncer do colo do útero, pois as mulheres não apresentaram queixa clínica, nem mesmo nas lesões potencialmente precursoras (HSIL).

Em um estudo feito com mulheres em tratamento por câncer do colo do útero, mostra que o primeiro sinal relatado pela maioria das pacientes é o sangramento vaginal, indo desde um pequeno fluxo até mesmo uma hemorragia severa, o qual foi o motivo da procura por atendimento médico, e as mesmas relataram a não realização dos exames preventivos quando não tinham esses sinais ou sintomas. A falta de informação e esclarecimento sobre esse câncer justifica a demora pela procura médica e como consequência o diagnóstico, visto que a fase inicial da doença é assintomática (PIMENTEL et al., 2011).

Considerando que a maioria das mulheres apresentaram o colo do útero normal durante a inspeção do colo cervical, e em média apenas $15,34 \%$ das mulheres tinham colo alterado, esses dados corroboram com a informação que a grande maioria das mulheres (90,37\% em média) não apresenta sangramento anormal, nem após relação sexual ou menopausa. Isso pode levar a mulher a não realizar periodicamente seu exame, seja pela ausência dos sintomas, ou pelo aspecto clínico normal do seu colo do útero.

\section{Conclusão}

Os dados evidenciados neste trabalho demostram a importância da realização do exame preventivo do câncer do colo do útero, pois na maioria das lesões intraepiteliais, as mulheres não apresentaram sintomas ou alterações clínicas. Espera-se que essas informações possam auxiliar os gestores nas estratégias de prevenção dessa patologia, pois conforme demostrado nesse trabalho, a maioria das mulheres com lesões potencialmente precursoras estão realizando seu exame de rotina, o que pode causar demora no seguimento dos exames complementares nessa população.

\section{Referências}

BACKERS, L. T. H. et al. Alterações citológicas cervicovaginais no Alto Uruguai Gaúcho, Rio Grande do Sul. Revista Ciências Médicas, v. 23, n. 2, p. 65-73, 2014.

BORTOLON, P. C. et al. Avaliação da Qualidade dos Laboratórios de Citopatologia do Colo do Útero no Brasil. Revista Brasileira de Cancerologia, v. 58, n. 3, p. 435444, 2012.

BRASIL. Ministério da Saúde. Avaliação de Indicadores das Ações de Detecção Precoce dos Cânceres do Colo do Útero e de Mama - Brasil e Regiões, 2013. Instituto Nacional do câncer José de Alencar Gomes da Silva. Coordenação Geral de Prevenção e Vigilância. Divisão de Detecção Precoce e Apoio à Organização de Rede. Rio de Janeiro. 2015.

BRASIL. Ministério da Saúde. Controle dos cânceres do colo do útero e da mama / Ministério da Saúde, Secretaria de Atenção à Saúde, Departamento de Atenção Básica. - 2. ed. - Brasília: Editora do Ministério da Saúde, 2013. 124 p.: il. (Cadernos de Atenção Básica, n. 13).

BRASIL. Ministério da Saúde. Diretrizes brasileiras para o rastreamento do câncer do colo do útero / Instituto Nacional de Câncer. Coordenação Geral de Ações Estratégicas. Divisão de Apoio à Rede de Atenção Oncológica. - Rio de Janeiro: INCA, 2011.

BRASIL. Ministério da Saúde. Estimativa 2016: incidência de câncer no Brasil / Instituto Nacional de Câncer José Alencar Gomes da Silva - Rio de Janeiro: INCA, 2015.

BRASIL. Ministério da Saúde. Informativo Detecção precoce. Boletim ano 5 n.1. janeiro/abril 2014. Instituto Nacional de Câncer José Alencar Gomes da Silva/ Ministério da Saúde.

BRASIL. Ministério da Saúde. Manual de gestão da qualidade para laboratório de citopatologia/ Instituto 
Nacional de Câncer José Alencar Gomes da Silva, Coordenação-Geral de Prevenção e Vigilância, Divisão de Detecção Precoce e Apoio à Organização de Rede. - Rio de Janeiro: Inca, 2012.

BRASIL. Ministério da Saúde. Nomenclatura brasileira para laudos citopatológicos cervicais / Instituto Nacional de Câncer José Alencar Gomes da Silva, CoordenaçãoGeral de Prevenção e Vigilância, Divisão de Detecção Precoce e Apoio à Organização de Rede. - 3. ed. - Rio de Janeiro: Inca, 2012.

\section{BRASIL. PRESIDÊNCIA DA REPÚBLICA. Portal} do Planalto. Em Manaus, Presidenta Dilma lança a campanha de combate ao câncer de mama e colo do útero. 2013. Disponível em: <http://www2.planalto.gov. br/acompanhe-o-planalto/releases/em-manaus-presidentadilma-lanca-a-campanha-de-combate-ao-cancer-de-mamae-colo-do-utero>. Acesso em: 20 ago. 2016.

\section{FIGUEREDO, E. N. A Estratégia Saúde da Família na} Atenção Básica do SUS. 2012. Disponível em: <http:// www.unasus.unifesp.br/biblioteca_virtual/esf/2/unidades conteudos/unidade05/unidade05.pdf $>$ Acesso em 02 set. 2016.

Instituto Nacional do Câncer José Alencar Gomes da SilvaINCA. Agenda Estratégica para o Controle do Câncer do Colo do Útero. 2015. Disponível em: < http://www2. inca.gov.br/wps/wcm/connect/acoes_programas/site/home/ agenda_estrategica/apresentacao_agenda_estrategica $>$ Acesso em: 25 ago. 2016.

Instituto Nacional do Câncer José Alencar Gomes da SilvaINCA. Atlas On line de Mortalidade. Disponível em: $<$ https://mortalidade.inca.gov.br/MortalidadeWeb/>. Acesso em: 25 ago. 2016 .

Instituto Nacional do Câncer José Alencar Gomes da Silva-INCA. Colo do Útero - Detecção Precoce. 2015. Disponível em. <http:/www2.inca.gov.br/wps/wcm/ connect/tiposdecancer/site/home/colo_utero/deteccao precoce $>$. Aceso em: 26 ago. 2016.

Instituto Nacional do Câncer José Alencar Gomes da SilvaINCA. Controle do Câncer do Colo do Útero: Conceito e Magnitude. 2015. Disponível em: <http://www2.inca.gov. br/wps/wcm/connect/acoes_programas/site/home/nobrasil/ programa_nacional_controle_cancer_colo_utero/conceito magnitude>. Acesso em: 26 ago. 2016.

Instituto Nacional do Câncer José Alencar Gomes da SilvaINCA. Controle do Câncer do Colo do Útero: Detecção Precoce. 2015. Disponível em: <http://www2.inca.gov.br/ wps/wcm/connect/acoes_programas/site/home/nobrasil/ programa_nacional_controle_cancer_colo_utero/deteccao_ precoce>. Acesso em: 30 ago. 2016

LAGANÁ, M. T. C. et al. Alterações Citopatológicas, Doenças Sexualmente Transmissíveis e Periodicidade dos Exames de Rastreamento em Unidade Básica de Saúde.

Revista Brasileira de Cancerologia, v. 59, n. 4, p. 523-
$530,2013$.

MANRIQUE, E. J. C. et al. Fatores que Comprometem a Adequabilidade da Amostra Citológica Cervical. Femina, vol. 37, n. 5, maio/2009.

MOURA, A. D. A. et al. Conhecimentos e Motivações das Mulheres Acerca do Exame Papanicolaou: Subsídios para a Prática de Enfermagem. Revista Rene, v. 11, n. 1, p. 94$104,2010$.

OLIVEIRA, I. S. B. et al. Ações das Equipes de Saúde da Família na Prevenção e Controle do Câncer de Colo de Útero. Revista Ciências, Cuidado e Saúde, v. 9, n. 2, p. 220-227, 2010.

PIMENTEL, A. V. et al. A Percepção da Vulnerabilidade Entre Mulheres com Diagnóstico Avançado do Câncer do Colo do Útero. Texto\& Contexto - Enfermagem. Florianópolis, v. 20, n. 2, p. 255-262, 2011.

Portal Brasileiro de Dados Abertos. Unidades Básicas de Saúde. 2016. Disponível em: < http://dados.gov.br/dataset/ unidades-basicas-de-saude-ubs $>$. Acesso em: 02 set. 2016.

PRADO, P. R. et al. Caracterização do Perfil das Mulheres com Resultado Citológico ASCUS/AGC, LSIL e HSIL segundo Fatores Sóciodemográficos, Epidemiológicos e Reprodutivos em Rio Branco-AC, Brasil. Revista Brasileira de Cancerologia, v. 58, n. 3, p. 471-479, 2012.

ROCHA, B. D. et al. Exame Papanicolaou: Conhecimento de Usuárias de uma Unidade Básica de Saúde. Revista de Enfermagem da UFSM, v. 2, n. 3, p. 619-629, 2012.

Secretaria Municipal de Saúde de Cascavel-PR - SESAU. Unidades de Saúde. 2015. Disponível em: <http://sesau. cascavel.pr.gov.br/unidadesdesaude.html $>$ Acesso em: 02 set. 2016.

SILVA, E. O.; COELHO, M. C. V.; ATHAYDE, L. A. Alterações Citológicas Associadas a Infecção pelo Pamilomavírus Humano em Mulheres Atendidas em um Hospital. Revista Eletrônica Gestão \& Saúde, Vol. 07, № 01, p. $52-64,2016$.

SILVA, D. S. M. et al. Rastreamento do Câncer do Colo do Útero no Estado do Maranhão, Brasil. Revista Ciência \& Saúde Coletiva, v. 19, n. 4, p. 1163-1170, 2014.

World Health Organization-WHO. Human papillomavirus (HPV) and cervical cancer. 2016. Disponível em: <http:// www.who.int/mediacentre/factsheets/fs380/en/>. Acesso em: 02 set. 2016 . 